\title{
Post-Anthesis Moisture Increased Fusarium Head Blight and Deoxynivalenol Levels in North Carolina Winter Wheat
}

\author{
Christina Cowger, Jennifer Patton-Özkurt, Gina Brown-Guedira, and Leandro Perugini
}

First and second authors: United States Department of Agriculture-Agricultural Research Service (USDA-ARS), Department of Plant Pathology, and third author: USDA-ARS, Department of Crop Science, North Carolina State University, Raleigh, NC 27695; and fourth author: Pioneer Hi-Bred, 985 County Road 300 E., Ivesdale, IL 61851.

Accepted for publication 29 December 2008.

\begin{abstract}
Cowger, C., Patton-Özkurt, J., Brown-Guedira, G., and Perugini, L. 2009. Post-anthesis moisture increased Fusarium head blight and deoxynivalenol levels in North Carolina winter wheat. Phytopathology 99:320-327.

Current models for forecasting Fusarium head blight (FHB) and deoxynivalenol (DON) levels in wheat are based on weather near anthesis, and breeding for resistance to FHB pathogens often relies on irrigation before and shortly after anthesis to encourage disease development. The effects of post-anthesis environmental conditions on FHB are poorly understood. We performed a field experiment at Kinston, NC, to explore the effects of increasing duration of post-anthesis moisture on disease incidence, disease severity, Fusarium-damaged kernels (FDK), percent infected kernels, and DON. The experiment had a split-plot design, and one trial was conducted in each of two successive years. Main plots consisted of post-anthesis mist durations of $0,10,20$, or 30 days. Subplots were of eight cultivars in the first year and seven in the second year, two being susceptible to FHB and the remainder each with varying degrees of apparent type I and type II resistance. Plots were inoculated by

the same effect $(P \geq 0.198)$ and were associated with an approximately fourfold increase in mean disease incidence and eightfold increase in disease severity compared with 0 days of mist $(P \leq 0.0002)$. In both years, mean FDK percentages at 0 and 10 days post-anthesis mist were the same and significantly lower than FDK percentages under 20 or 30 days of post-anthesis mist. Mist duration had a significant effect on percent kernels infected with Fusarium spp. as detected by a selective medium assay of 2007 samples. Averaging across all cultivars, in both years, DON levels increased significantly for 10 days compared with 0 days of mist, and increased again with 20 days of mist $(P \leq 0.04)$. This is the first investigation to show that extended post-flowering moisture can have a significant enhancing effect on FHB, FDK, DON, and percent infected kernels of wheat. For all disease and toxin assays, cultivar rankings were significantly noncorrelated among mist durations in at least 1 year, suggesting that FHB screening programs might rank genotypes differently under extended post-anthesis moisture than without it. Our findings also imply that accurate forecasts of DON in small grains must take account of post-anthesis weather conditions.
\end{abstract} spraying Fusarium graminearum macroconidia at mid-anthesis. Averaging across years and cultivars, 10 or 20 days of post-anthesis mist had
Additional keywords: Gibberella zeae.
Gibberella zeae (anamorph Fusarium graminearum) is the most important causal agent of Fusarium head blight (FHB) (or scab) of wheat and barley in the United States, and is a pathogen of global importance $(14,22)$. FHB lowers grain yield and test weight and contaminates grain with mycotoxins such as deoxynivalenol (DON). DON and other tricothecene mycotoxins are hazards to mammalian health (24) and are a major determinant of fungal spread and disease development in the Triticeae (10). The toxins can be translocated within wheat heads via the xylem and phloem and can accumulate in cells in advance of the pathogen (9).

Among the factors determining DON levels in wheat are distinct host resistance types, which are under separate genetic control $(15,16,25,26)$. Type I resistance, or resistance to initial Fusarium infection (26), appears to be related to increased activity of lignification-associated compounds (17,27); other factors may also be involved. Type II resistance, or resistance to disease spread within a spike, is correlated with resistance to DON in wheat heads (12), and may be governed by the same quantitative trait loci (QTL) as DON resistance (2). Antifungal compounds have also been implicated in FHB resistance that is

Corresponding author: C. Cowger; E-mail address: Christina.Cowger@ars.usda.gov

doi:10.1094/PHYTO-99-4-0320

This article is in the public domain and not copyrightable. It may be freely reprinted with customary crediting of the source. The American Phytopathological Society, 2009. postulated to be type II $(6,23)$. Some cultivars, such as Frontana, possess both type I and type II resistance, whereas other cultivars appear to have only one type $(26,27)$. Pyramiding of alleles conferring resistance types I and II is widely sought (25) and can lead to disease reductions of as much as $50 \%$ (1).

In the United States, wheat grain with DON concentrations exceeding U.S. Food and Drug Administration (FDA) advisory levels may be rejected or devalued at grain intake points $(3,14)$. Growers and grain purchasers benefit from accurate predictions as to whether DON in harvested grain will exceed acceptable levels. Models have been developed to forecast the probability of an FHB epidemic of $>10 \%$ severity (5) or to forecast DON concentrations in mature grain (8). Because of the need for forecasts that are timely for fungicide decisions, these models are based on weather variables from approximately 12 days prior to anthesis to 10 days post-anthesis.

However, a study of the 2003 FHB epidemic in the Southeast (3) suggested that FHB severity and DON levels in this case may have been more strongly influenced by weather later in the grainfill period. The influence of post-anthesis weather variables such as moisture on disease and DON levels is still not well understood. In the few studies of this topic, experimental protocols have varied considerably. In a Minnesota field study using spring wheat, Culler et al. (4) subjected two moderately resistant cultivars and one susceptible cultivar to two durations of post-anthesis misting, 15 to 16 days versus 31 to 32 days. They found that DON concentrations from early dough through harvest were lower under the longer misting duration, which they hypothesized 
might be due to leaching of DON. Lemmens et al. (11) planted 10 winter wheat cultivars and inoculated them every other day for over 2 weeks in an Austrian field experiment. In treatments irrigated throughout the inoculation period and for another 26 days, mean disease severity was higher but DON concentrations were lower than for nonirrigated treatments.

Lemmens et al. (11) also found an interaction between host genotype and irrigation with respect to DON accumulation. That is, some genotypes reacted to extended moisture with a decrease in DON content, while others had increased DON content. The authors hypothesized that the appearance of "premature spikes" (those with the upper portion wilted, leading to bleaching and yield reduction, but not colonized by the fungus) could explain the combination of an increase in disease symptoms, a reduction in yield, and a reduction in grain DON.

Controlled studies are needed to investigate the relative importance of post-anthesis weather on FHB severity and DON levels. In the present investigation, our goal was to better understand the effect of different numbers of post-anthesis wet days on FHB severity and DON accumulation in wheat grain. We hypothesized that increasing durations of moisture would be associated with elevated disease symptoms and DON concentrations. We planted field plots of soft red winter wheat cultivars adapted to southeastern U.S. growing conditions and possessing varying levels and apparent types of FHB resistance. The cultivars were inoculated at mid-anthesis and plots were subjected to durations of post-anthesis mist from 0 to 30 days in order to capture a wider range of wet-day durations than those found in previous studies. The data we acquired on disease, Fusarium-damaged kernels (FDK), percent kernels infected, and DON allowed us to examine the relationship of post-anthesis moisture to visual symptoms, DON development, and kernel damage.

\section{MATERIALS AND METHODS}

Field experiment location and design. The field experiment was conducted in a misted nursery at the Cunningham Research and Extension Center in Kinston, NC. One trial was performed in each of two field seasons, 2005-06 and 2006-07, which will hereafter be referred to as 2006 and 2007, respectively. Both trials were planted in conventionally tilled fields (full primary and secondary tillage, burying residues) following corn or soybean.

The experiment had a split-plot design, with duration of postflowering moisture as the main plot and cultivar as the subplot. The main-plot irrigation regimes were $0,10,20$, and 30 days of post-anthesis misting. Three replicate plots of each cultivar were subjected to each duration of post-anthesis misting, and cultivars were randomly assigned to subplots within moisture duration main plots. Plots were planted with a five-row plot drill. After end trimming, each plot measured $3.1 \mathrm{~m}$ in length and $1.2 \mathrm{~m}$ in width.

Cultivars for the experiment (Table 1) were chosen from among regionally adapted lines with similar maturities, and so as to have a variety of putative resistance types (type I, or resistance to primary infection; type II, or resistance to subsequent colonization; or both) (15). For the moderately resistant cultivars, resistance types were postulated based on incidence (percentage of diseased spikes), severity (percentage of diseased spikelets), and DON data from the 2000 to 2003 Uniform Southern Soft Red Winter Wheat FHB Screening Nurseries (18-21). As a crude indicator of resistance type, a high incidence-to-severity ratio was taken as evidence of type II resistance and, conversely, a high severity-toincidence ratio as evidence of type I resistance. Cultivars had appeared in the uniform nursery for varying numbers of years. VA01W-99 was an elite line supplied by J. Chen and C. Griffey, Virginia Polytechnic University, with pedigree FFR525/93-52-55 (Massey*3/Balkan//Saluda), that had shown a high level of FHB resistance in the field.

According to the results of assays with 42 simple-sequence repeat markers, and as expected based on pedigrees, none of the cultivars examined was judged to have either the type I resistance QTL on chromosome 5AS that is present in Sumai 3 or the type II resistance QTL on chromosomes 2DL (Wuhan 1), 3BS (Sumai 3), 4B (Wuhan 1), and 6BS (Sumai 3) (13) (data not shown). Type II resistance loci mapped in the soft winter wheat cv. Ernie in the 3BSc and $4 \mathrm{~B}$ regions did not appear to be present in the other cultivars in this study (data not shown). The marker haplotype of Ernie in the 3BSc and 4B regions was unique in this set of eight soft wheat cultivars and did not match the haplotypes of Wuhan 1 or Sumai 3 (data not shown). Marker haplotypes for the 5AL region in cvs. NC Neuse, Vigoro-Tribute, and VA01W-99 differed from the Ernie haplotype, although VA01W-99 shared a partial haplotype (three of four marker alleles) with Ernie (data not shown).

Inoculation and misting. To generate inoculum for the field experiment, four isolates of $F$. graminearum with proven pathogenicity to wheat (C. Cowger, unpublished data) were increased individually in aerated 20-liter carboys of Mung bean tea. The tea was prepared by steeping Mung beans in boiled $\mathrm{dH}_{2} \mathrm{O}$ at a rate of $40 \mathrm{~g} /$ liter for $15 \mathrm{~min}$, filtering the tea through cheesecloth, and autoclaving. Carboys containing 12 liters of tea were spiked with $500 \mathrm{ml}$ of tea in which macroconidia had been previously grown, and then aerated with sterile air for approximately 1 week before centrifugation at 4,000 rpm for $3 \mathrm{~min}$.

TABLE 1. Soft red winter wheat cultivars planted in Kinston, NC, field experiment on the effects of post-anthesis moisture on Fusarium head blight (FHB) severity and deoxynivalenol accumulation

\begin{tabular}{|c|c|c|c|c|c|c|c|}
\hline \multirow[b]{3}{*}{ Treatment } & \multirow[b]{3}{*}{ P.I./PVP number ${ }^{u}$} & \multirow[b]{3}{*}{ Date $(\text { Julian })^{\mathrm{v}}$} & \multirow[b]{3}{*}{$\mathrm{FHB}^{\mathrm{w}}$} & \multirow[b]{3}{*}{ Type $^{\mathrm{x}}$} & \multicolumn{3}{|c|}{ Greenhouse experiments (\% infected spikelets) ${ }^{\mathrm{y}}$} \\
\hline & & & & & \multirow{2}{*}{$\frac{\text { Type I resistance }}{2 \text { trials }}$} & \multicolumn{2}{|c|}{ Type II resistance } \\
\hline & & & & & & 2 trials & 4 trials \\
\hline NC Neuse & 200400303 & 115 & MR & II & $16.1 \mathrm{~b}$ & $41.3 \mathrm{ab}$ & $32.1 \mathrm{ab}$ \\
\hline Ernie & 9600360 & 109 & MR & I & $2.0 \mathrm{a}$ & $33.0 \mathrm{ab}$ & $35.1 \mathrm{ab}$ \\
\hline VA01W-99 & Experimental & 111 & MR & II & $8.5 \mathrm{ab}$ & $54.0 \mathrm{bc}$ & $39.6 \mathrm{ab}$ \\
\hline Vigoro Tribute $^{\mathrm{z}}$ & 632689 & 112 & MR & I & $\ldots$ & $55.9 \mathrm{bc}$ & $\ldots$ \\
\hline USG 3592 & 200400110 & 112 & S & Susc. & $29.3 \mathrm{c}$ & $71.2 \mathrm{c}$ & $76.4 \mathrm{c}$ \\
\hline
\end{tabular}

" P.I. = plant introduction and PVP = plant variety protection.

${ }^{v}$ Heading date (Julian), based on 2005 data from the Cunningham Research Station OVT and the author's experiment.

${ }^{\text {w }}$ FHB resistance, based on 2005 field observations and 2002-2003 data from USSRWWFHBSN. MR = moderately resistant and S = susceptible.

${ }^{x}$ Putative resistance type (I, II, or susceptible [Susc.]), based on incidence: severity ratio in 2000-2003 USSRWWFHBSN data, except VA01W-99.

y Two trials of spray inoculation (type I) in 2007, scored as percentage of diseased spikelets at 7 days after inoculation (dai), and two trials of point inoculation (type II) in each of 2006 and 2007, with percentage of diseased spikelets at 14 dai. Within a column, means followed by the same letter are not significantly different at $P \leq 0.05$.

z Only planted in the field experiment in 2006 (seed contamination in 2007). 
For inoculation of field plots, a suspension of $10^{5}$ macroconidia/ml was used. The suspension was composed of equal proportions of spores of the four isolates, and plants in the plots were inoculated at mid-anthesis (Feekes growth stage 10.5.1 to 10.5.2) using a backpack sprayer. Mid-anthesis was considered to be the day on which $50 \%$ of the heads of a given cultivar had extruded anthers; thus, cultivars were inoculated on different dates within a season. The dates of inoculation were 13 to 19 April 2006 and 17 to 25 April 2007. Determining mid-anthesis was complicated in 2007 by the patchy appearance of Soilborne wheat mosaic virus (SBWMV) within the nursery, which caused mild stunting and retardation of anthesis in some plots.

Misting was provided using Roberts 435/436 sprinkler heads, which have small-diameter $(1.4-\mathrm{mm})$ orifices, mounted on risers at a height of $0.91 \mathrm{~m}$ above the ground. The different durations of post-anthesis mist were provided by opening or closing individual lateral irrigation lines. By means of a programmable timer, mist was provided for $2 \mathrm{~min}$ of each 20 -min period for $3 \mathrm{~h}$ each in the morning and afternoon (0800 to 1100 and 1400 to $1700 \mathrm{~h}$ ), or a total of 36 min per day. Taking sprinkler overlap into account, actual flow delivered was $0.275 \mathrm{~mm} / \mathrm{min}$. All periods of postanthesis mist $(0,10,20$, or 30 days) started on 17 April and 23 April in the two years, respectively.

Due to limited misted nursery space, buffer plots could not be planted between all treatments. However, buffer passes of the moderately resistant $\mathrm{cv}$. NC Neuse were inserted to avoid interference of irrigation treatments, and borders of NC Neuse were planted around the experiment.

Disease assessment, sample collection, and FDK and DON assays. In each year, disease assessments were conducted on each cultivar approximately 20 days after it had been inoculated. The dates of these assessments were 4 to 9 May 2006 and 7 to 15 May 2007. Disease incidence (DI) and disease severity (DS) were recorded in all plots by assessing 10 consecutive main heads at each of four randomly chosen locations within the plot. DI was determined as the percentage of heads with diseased spikelets and DS as the mean percentage of diseased spikelets in the total of 40 heads. A given replicate was assessed by the same observer across all main plots and subplots. The outside rows and the terminal $0.3-\mathrm{m}$ portions of plots were avoided in order to minimize edge effects. In 2007, cv. VA01W-99 was excluded from DI and DS assessments due to the effects of a late spring freeze.

Spike samples were hand harvested on 9 June 2006 and 7 June 2007, which was the normal commercial harvest time for winter wheat in North Carolina. Samples consisted of 100 randomly chosen spikes per plot. Again, in order to minimize interplot interference, samples were not gathered from the outer rows or the terminal $0.3-\mathrm{m}$ portions of plots.

Spikes were threshed in a single-head thresher (Precision Machine, Lincoln, NE), such that all kernels were retained. To determine the percentage of FDK, 100 kernels were chosen at random from the yield of each plot and visually inspected for gray-white or pink discoloration and mycelial growth. Eighty-gram samples of grain were sent for determination of DON content using enzyme-linked immunosorbent assay analysis at the Regional Diagnostic Clinic for Fusarium Head Blight at Michigan State University in 2006, and by Dr. Yanhong Dong at the University of Minnesota using gas chromatography-mass spectrometry in 2007.

Data on daily precipitation for the experimental periods were obtained from the North Carolina State Climate Office weather station at the Cunningham Research and Extension Center.

Greenhouse assays. To further characterize the type of resistance present in the cultivars used in the field, each cultivar was assayed for type I and type II resistance in the greenhouse. The cultivars were also subjected to molecular marker assays for type I and type II resistance QTL.

The greenhouse trials were conducted in the February to April periods of 2006 and 2007. In both years, the eight cultivars in
Table 1 were tested in all trials; however, the 2007 trials utilized contaminated seed for cv. Tribute, and those data are omitted. Two trials using spray inoculation (for type I resistance) were conducted in 2007. Two trials using point inoculation (for type II resistance) were conducted in each year, for a total of four type II trials. For each trial of both techniques, one vernalized seedling of each cultivar was planted in each Deepot (Stuewe \& Sons, Corvallis, OR). There were five replicate pots of each cultivar in each trial in the first year and nine replicate pots of each cultivar in each trial in the second year. In all trials of both techniques, inoculum consisted of a water suspension of one of three of the $F$. graminearum isolates used in the field experiment. In the first year, individual isolates were applied to varying numbers of pots per cultivar; in the second year, each of the three isolates was applied to each of three pots per cultivar in each trial. Analysis of variance of the results indicated that there were no significant differences among the three isolates in the amount of disease caused $(P \geq 0.253)$. In all trials, plants were placed in a mist chamber for $72 \mathrm{~h}$ after inoculation, and only the primary spike on each plant was included in the data analysis.

For type I assays, approximately $200 \mu$ l of a macroconidial $F$. graminearum solution at a concentration of $10^{5}$ spores $/ \mathrm{ml}$ was sprayed at early anthesis on the main spike in each pot using an atomizer. The percentage of symptomatic spikelets was assessed at 7 days after inoculation (dai), when each visibly diseased spikelet was assumed to be the result of a separate infection event, and spread from initially infected spikelets was not yet evident. Type I resistance was taken to be (diseased spikelets)/(total number of spikelets) $\times 100$ at 7 dai.

Type II resistance was assessed via pipette inoculations performed at early anthesis, using $10 \mu$ of spore solution at $5 \times$ $10^{4}$ spores $/ \mathrm{ml}$ and inoculating a single central spikelet (25). The percentage of diseased spikelets in each head was recorded at 7 , 14 , and 21 dai. Data analyzed were the 14-dai observations, which gave the best separation of the cultivars.

Data analysis. Data were analyzed using PROC MIXED in SAS (SAS Institute, Cary, NC), with a standard model for a splitplot design. Fixed effects were misting duration, cultivar, year, and the interactions of misting duration with cultivar and year, respectively. The replicate was treated as a random effect. Inspection of residual plots indicated that homoscedasticity was maximized by leaving data on DI and percentage of infected kernels untransformed, log-transforming DS data, and square-root-transforming FDK and DON data.

When DI and DS data were analyzed across years, only cultivars used in both years were included in the analysis (Tribute and VA01W-99 were not available in 2007). Analyses of FDK, percent infected kernels, and DON for both years together were based on seven cultivars (cv. Tribute was excluded in 2007).

Using SAS PROC CORR, Spearman rank correlation analyses were performed on the means of DI, DS, FDK, percent kernel infection, and DON data. These analyses were used to determine whether different durations of post-anthesis mist ranked cultivars differently. Averaging across replicates, cultivars were given ranks based on each dependent variable (in turn) and, for each pair of mist durations, the hypothesis was tested that rankings were the same, with $P \leq 0.05$ as the threshold for significant ranking difference.

\section{RESULTS}

Greenhouse assays. Results for the greenhouse tests for type I and type II resistance are given in Table 1. In some cases, resistance types that were hypothesized for moderately resistant cultivars based on ratios of incidence to severity in uniform nursery experiments were supported by greenhouse tests. For example, the greenhouse data supported the hypothesis that Coker 9474 had 
high levels of both type I and type II resistance, and that Ernie had strong type I resistance.

DI and DS. The disease data in Figure 1 were collected in the field approximately 20 days post-anthesis. Thus, the plots destined to receive 30 days of post-anthesis mist had not yet received the final 10 days of mist when these data were recorded. Analyses of DI and DS did not include the 30-day mist treatments but their data are reported in Figure 1 to facilitate interpretation of DON data from the same plots (reported below).

The three-way interaction of year, mist duration, and cultivar was significant (Table 2); therefore, disease results were analyzed separately by year. In each year, analyzed individually, the effect of mist duration on both DI and DS depended on cultivar $(P \leq$ 0.04). In 2006, all eight cultivars exhibited significantly higher DI and DS in response to moisture compared with the nonmisted control $(P \leq 0.05)$. In 2007 , five of six cultivars showed a significant DI and DS response to moisture $(P \leq 0.05)$; USG 3592 did not. Thus, although there was a significant interaction of mist duration with cultivar, cultivars generally exhibited increased FHB symptoms as moisture duration increased (Table 3). Similarly, the effect of mist duration on DI and DS depended on year (Table 2) but in both years there was an increase in disease in response to moisture (Table 3). Linear contrasts to dissect the interaction of cultivar susceptibility (moderately resistant [MR] versus susceptible $[\mathrm{S}]$ ) with mist duration indicated no significant interaction for incidence $(P=0.572)$, and yielded a $P$ value of
0.055 for severity. This suggests that the significant interaction of cultivar and mist duration was not primarily the result of moderate resistance being "overcome" under misting.

Using Spearman's rank correlation coefficient, DS rankings of cultivars were correlated among different post-anthesis mist durations in 2006 but not in 2007 (Table 3). DI rankings were also correlated among mist durations in 2006 but not in 2007 (data not shown, because the relationships are similar to those for DS).

Averaging across years and cultivars, 10 or 20 days of postanthesis mist were associated with higher mean DI and DS compared with 0 days $(P \leq 0.0002)$, and there was no difference in either DI or DS when comparing 10 with 20 days of mist $(P \geq$ 0.198). Averaging across cultivars, applying post-anthesis mist was associated with approximately 3- and 4-fold increases in DI in 2006 and 2007, respectively, and with approximately 4- and 13fold increases in DS in the 2 years, respectively (Table 3; Fig. 1).

Mean disease levels were higher in 2006 than in 2007 (Table 2; Fig. 1). This may be in part because the period between inoculation and disease assessment was wetter in 2006 than in 2007: there were $82.3 \mathrm{~mm}$ of rain between 17 April and 7 May 2006 but only $33.3 \mathrm{~mm}$ of rain between 23 April and 13 May 2007. Another cause was likely also SBWMV. For the two susceptible cultivars, DI was lower in 2007 than in 2006 whereas DS was similar in both years (Fig. 1).

Kernel damage and infection. Percentages of FDK were higher in 2006 than in 2007 (Tables 2 and 4). As with DI and DS,
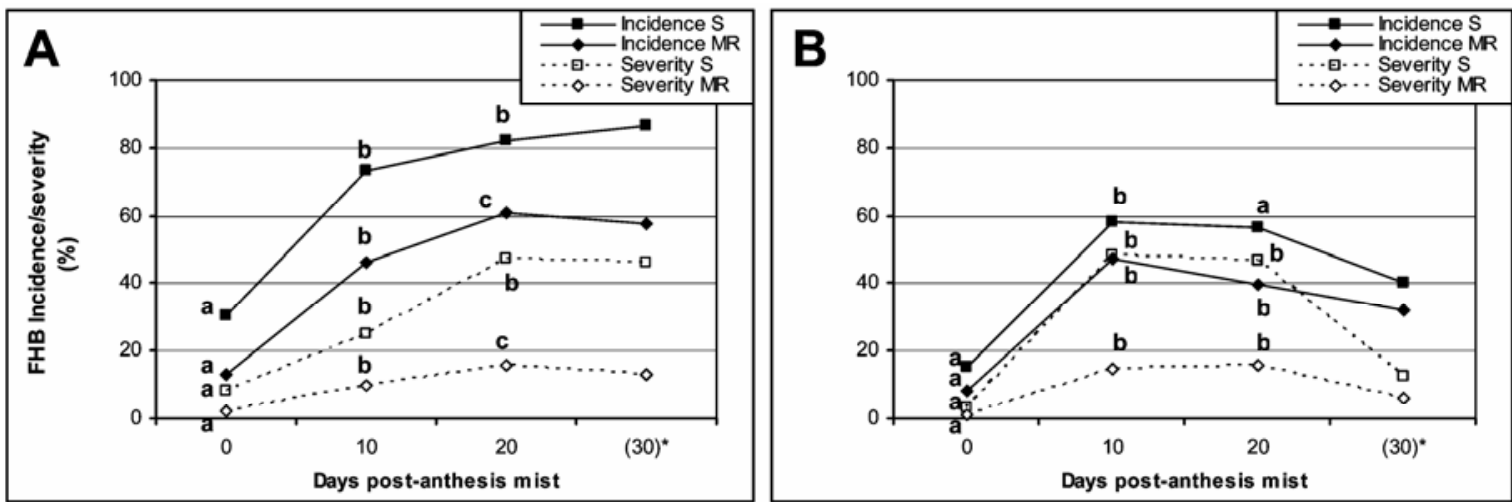

Fig. 1. Fusarium head blight incidence and severity in a misted field nursery at Kinston, NC, in trials conducted in A, 2005-06, where data are means of six moderately resistant (MR) and two susceptible (S) winter wheat cultivars, and B, 2006-07, data are means of four MR and two $S$ cultivars. Mist was applied for intervals of $0,10,20$, or 30 days, starting at mid-anthesis. Within a combination of year and resistance level (e.g., $2006 \mathrm{~S}$ ), data points with the same letter are not significantly different at $P \leq 0.05$. There were three replicate plots of each combination of cultivar and mist duration. *Note that disease assessments were conducted at approximately 20 days post-anthesis; thus, incidence and severity in plots destined for 30 days of post-anthesis mist were actually only affected by 20 days post-anthesis mist.

TABLE 2. Analysis of variance of Fusarium head blight (FHB) incidence, FHB severity, Fusarium-damaged kernels (FDK), and deoxynivalenol (DON) in soft red winter wheat cultivars subjected to varying durations of post-anthesis mist in a Kinston, NC, study of effects of post-anthesis moisture on FHB severity and DON accumulation

\begin{tabular}{|c|c|c|c|c|c|c|c|c|c|}
\hline \multirow[b]{2}{*}{ Source of variation } & \multirow[b]{2}{*}{$\mathrm{df}^{\mathrm{w}}$} & \multicolumn{2}{|c|}{ Disease incidence } & \multicolumn{2}{|c|}{ Disease severity } & \multicolumn{2}{|c|}{ FDK $^{\mathrm{x}}$} & \multicolumn{2}{|c|}{$\mathrm{DON}^{\mathrm{x}}$} \\
\hline & & $F$ & $P$ & $F$ & $P$ & $F$ & $P$ & $F$ & $P$ \\
\hline Year & 1 & 16.6 & 0.0001 & 10.6 & 0.002 & 257.7 & $<0.0001$ & 461.6 & $<0.0001$ \\
\hline Mist $^{\mathrm{y}}$ & $2 / 3$ & 52.2 & 0.0002 & 66.7 & $<0.0001$ & 50.3 & $<0.0001$ & 68.2 & $<0.0001$ \\
\hline Year × cv. & $5 / 6$ & 14.7 & $<0.0001$ & 11.1 & $<0.0001$ & 2.3 & 0.044 & 7.7 & $<0.0001$ \\
\hline Year $\times$ mist & $2 / 3$ & 5.0 & 0.010 & 3.4 & 0.038 & 7.5 & 0.0001 & 10.2 & $<0.0001$ \\
\hline Cv. $\times$ mist & $10 / 18$ & 2.8 & 0.006 & 3.6 & 0.001 & 2.1 & 0.009 & 1.5 & 0.100 \\
\hline
\end{tabular}

${ }^{\mathrm{w}}$ First number applies to disease incidence (DS) and disease severity (DS); second number applies to FDK and DON.

${ }^{x} F$ and $P$ values are from analysis using square-root-transformed data to increase homoscedasticity.

${ }^{y}$ Mist duration. Disease incidence and severity were analyzed for plots misted 0, 10, or 20 days post-anthesis; 30-day misted treatments were omitted because disease was assessed in all plots at approximately 20 days post-anthesis.

${ }^{z}$ Six cultivars were evaluated for DI and DS in both years: NC Neuse, Coker 9474, USG 3650, and Ernie, which were moderately resistant; and Coker 9184 and USG 3592, which were susceptible. In addition to those cultivars, a seventh cultivar (VA01W-99, which was moderately resistant) was evaluated for FDK and DON in both years. 
the strongest effects on FDK were of year and mist duration, although the interactions of those factors with each other and with cultivar were significant (Table 2). In both years, FDK percentages at 0 and 10 days post-anthesis were the same and were significantly lower than FDK percentages under 20 or 30 days of mist (Table 4). In the 2 years, seven of the eight cultivars and two of the seven cultivars, respectively, showed a significant $(P \leq$ 0.05) FDK response to mist (data not shown). In both years, cultivar FDK rankings were significantly uncorrelated among mist durations (Table 4).

Kernel-infection assays conducted on the 2007 samples using Komada's medium yielded a similar picture (Table 5). Analysis of variance (data not shown) indicated that mist duration had a significant effect $(F$ value $=51.2, P<0.0001)$ on percent kernels infected. The effect of cultivar was also significant $(F$ value $=9.1$, $P<0.0001)$, as was the cultivar $\times$ mist duration interaction $(F$ value $=2.1, P=0.024)$. All seven cultivars showed a significant response to mist duration $(P \leq 0.05)$. By Spearman's rank correlation analysis, cultivar rankings for percentage of infected seed were different under 10 days of post-anthesis mist than those under 0,20 , or 30 days of mist (Table 5).

DON. DON concentrations are presented in Table 6. Analysis of variance indicated significant effects on DON of mist duration, cultivar, and year (Table 2). The analysis also showed a significant interaction of year with misting duration (Table 2), likely owing to the different effect in the 30-day plots in the 2 years
(Fig. 2; Table 6). The interaction of mist duration and cultivar was significant; however, in each year, each cultivar showed a significant increase in DON in response to increasing mist duration $(P \leq 0.05)$.

Mean DON levels were higher in 2006 than in 2007 (8.8 versus $2.7 \mu \mathrm{g} / \mathrm{g}$ ) (Fig. 2). Mean DON concentrations for each year are shown in Figure 2, with the levels for $\mathrm{S}$ and MR cultivars depicted separately. In both years, plots receiving 20 days of post-anthesis mist had DON concentrations higher than those receiving 0 days mist for both S and MR cultivars (Table 6; Fig. 2). In 2006, DON concentrations were higher with 10 days of mist than with 0 days mist for MR cultivars but not for S cultivars (Fig. 2).

Averaging across cultivars, in both years DON levels increased significantly for 10 days compared with 0 days of mist, and increased again with 20 days of mist $(P \leq 0.04)$. In 2006 , DON from plots misted for 30 days was not different than DON from plots misted for 20 days $(P=0.95)$ whereas, in 2007 , DON was lower in 30-day than in 20-day misted plots $(P<0.0001)$ and was not different from DON in plots receiving 10 days of mist $(P=$ 0.306).

Combining DON data from the 2 years, there was no significant interaction of cultivar and misting duration (Table 2) (i.e., the effect of cultivar on DON did not change according to mist duration). When the years were analyzed separately, however, this interaction was significant $(P=0.01$ and 0.005 in 2006 and 2007, respectively). DON rankings did not differ significantly between

TABLE 3. Fusarium head blight severity and rank correlation results for soft red winter wheat cultivars subjected to varying durations of post-anthesis mist in a Kinston, NC, field experiment

\begin{tabular}{|c|c|c|c|c|c|c|c|c|c|c|c|c|}
\hline \multirow{3}{*}{ Cultivar } & \multicolumn{12}{|c|}{ Severity ${ }^{\mathrm{x}}$} \\
\hline & \multicolumn{6}{|c|}{ Days of post-anthesis mist, 2006} & \multicolumn{6}{|c|}{ Days of post-anthesis mist, 2007} \\
\hline & \multicolumn{2}{|c|}{0} & \multicolumn{2}{|c|}{10} & \multicolumn{2}{|c|}{20} & \multicolumn{2}{|c|}{0} & \multicolumn{2}{|c|}{10} & \multicolumn{2}{|c|}{20} \\
\hline Ernie & 1.0 & 1 & 1.9 & 1 & 6.4 & 1 & 3.0 & 5 & 31.0 & 5 & 4.4 & 2 \\
\hline Coker 9474 & 1.3 & 2.5 & 16.1 & 6 & 17.7 & 5 & 0.2 & 1 & 7.4 & 3 & 8.8 & 3 \\
\hline NC Neuse & 1.3 & 2.5 & 8.8 & 3 & 15.1 & 4 & 1.2 & 3 & 4.9 & 2 & 9.6 & 4 \\
\hline USG 3650 & 3.2 & 6 & 14.5 & 5 & 26.3 & 6 & 0.9 & 2 & 15.4 & 4 & 40.0 & 5 \\
\hline USG 3592 & 5.7 & 7 & 23.2 & 7 & 59.8 & 8 & 2.5 & 4 & 4.6 & 1 & 3.6 & 1 \\
\hline Coker 9184 & 11.0 & 8 & 27.1 & 8 & 34.9 & 7 & 4.3 & 6 & 92.4 & 6 & 90.1 & 6 \\
\hline Mean $^{z}$ & $3.9 \mathrm{a}$ & $\ldots$ & $15.3 \mathrm{~b}$ & $\ldots$ & $26.7 \mathrm{~b}$ & $\ldots$ & $2.0 \mathrm{a}$ & $\ldots$ & $26.0 \mathrm{~b}$ & $\ldots$ & $26.1 \mathrm{~b}$ & $\ldots$ \\
\hline
\end{tabular}

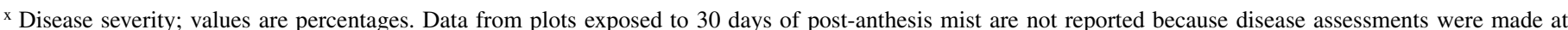
approximately 20 days post-anthesis. Within a year, two mist durations whose rank column headings are followed by the same uppercase letter did not rank cultivars differently at $P \leq 0.05$ by Spearman's rank correlation test. A lower rank number indicates less disease.

y V-Tribute was not planted in 2007 due to a seed mix-up, and VA01W-99 could not be assessed for disease due to a late spring freeze.

z Within a year, means followed by the same letter are not different at $P \leq 0.05$ by least-squares means.

TABLE 4. Fusarium-damaged kernel (FDK) percentages and rank correlation results for soft red winter wheat cultivars in inoculated North Carolina field experiment on the effects of post-anthesis moisture on Fusarium head blight (FHB) severity and deoxynivalenol (DON) accumulation ${ }^{\mathrm{x}}$

\begin{tabular}{|c|c|c|c|c|c|c|c|c|c|c|c|c|c|c|c|c|}
\hline \multirow[b]{3}{*}{ Cultivar } & \multicolumn{8}{|c|}{ Days of post-anthesis mist, 2006} & \multicolumn{8}{|c|}{ Days of post-anthesis mist, 2007} \\
\hline & \multicolumn{2}{|c|}{0} & \multicolumn{2}{|c|}{10} & \multicolumn{2}{|c|}{20} & \multicolumn{2}{|c|}{30} & \multicolumn{2}{|c|}{0} & \multicolumn{2}{|c|}{10} & \multicolumn{2}{|c|}{20} & \multicolumn{2}{|c|}{30} \\
\hline & $\%$ & Rank A & $\%$ & Rank B & $\%$ & Rank C & $\%$ & Rank AC & $\%$ & Rank A & $\%$ & Rank B & $\%$ & Rank C & $\%$ & Rank D \\
\hline NC Neuse & 3.3 & 2 & 5.3 & 5 & 12.7 & 2 & 18.0 & 4 & 1.3 & 5 & 1.3 & 6 & 2.3 & 5 & 3.7 & 3.5 \\
\hline Ernie & 4.0 & 3 & 2.3 & 1 & 22.3 & 5.5 & 17.0 & 3 & 2.7 & 6 & 0.3 & 1 & 1.7 & 3 & 3.7 & 3.5 \\
\hline VA01W-99 & 4.7 & 4 & 4.3 & 3.5 & 22.3 & 5.5 & 22.3 & 5 & 0.7 & 4 & 0.7 & 3 & 1.7 & 3 & 3.0 & 2 \\
\hline USG 3650 & 5.0 & 5 & 11.7 & 8 & 12.3 & 1 & 12.3 & 1 & 0.3 & 3 & 1.0 & 5 & 1.7 & 3 & 0.7 & 1 \\
\hline USG 3592 & 12.0 & 8 & 7.7 & 7 & 23.0 & 7 & 57.3 & 8 & 0.0 & 1.5 & 0.7 & 3 & 4.0 & 6 & 7.3 & 7 \\
\hline Mean $^{\mathrm{z}}$ & $6.5 \mathrm{a}$ & $\ldots$ & $5.7 \mathrm{a}$ & $\ldots$ & $20.2 \mathrm{~b}$ & $\ldots$ & $24.3 \mathrm{~b}$ & $\ldots$ & $1.2 \mathrm{a}$ & $\ldots$ & $0.9 \mathrm{a}$ & $\ldots$ & $3.3 \mathrm{~b}$ & $\ldots$ & $4.3 \mathrm{~b}$ & $\ldots$ \\
\hline
\end{tabular}

${ }^{\mathrm{x}}$ Within a year, two mist durations whose rank column headings are followed by the same uppercase letter did not rank cultivars differently at $P \leq 0.05$ by Spearman's rank correlation test. A lower rank number indicates less FDK.

${ }^{y}$ V-Tribute was not planted in 2007 due to a seed mix-up, and VA01W-99 could not be assessed for disease due to a late spring freeze.

${ }^{\mathrm{z}}$ Means followed by the same letter within this row are not different at $P \leq 0.05$ by least-squares means. 
20- and 30-day mist durations in either year (Table 2) and, in each year, both of these longer mist durations ranked cultivars differently than did the 0-day post-flowering mist treatment, according to Spearman's rank correlation test. Ten days of post-flowering mist ranked cultivars differently from 0 days of mist in 2006 but not in 2007.

\section{DISCUSSION}

This is the first investigation to show that increasing durations of post-flowering moisture can have a significant enhancing effect on FHB, FDK, DON, and percent infected kernels of wheat. The increase in disease and DON associated with post-anthesis mist occurred in 2 years and for cultivars of varying levels and types of resistance.

What mechanisms could underlie the association between increasing durations of post-flowering moisture and increasing FHB and DON? One possibility is that the higher relative humidity around spikes is conducive to fungal development. In this vein, Hart et al (7) concluded that increasing duration of spike wetness favored increased production of DON, but their experiments were conducted by bagging heads following inoculation at growth stages ranging from watery-ripe to early dough, their results are thus not directly comparable with ours. Another possible factor is reduced water stress in host plants, which could facilitate spike colonization or DON production, perhaps by delaying the onset of senescence.

In 2006, DON was the same in plots misted for 20 and 30 days, while in 2007, DON was lower in the 30-day misted plots (Fig.
1). However, this was likely an artifact because, in 2007, DI and DS disease levels were also lower in the 30-day plots, even though at disease assessment time both they and the 20-day plots had received 20 days of mist. The confounding effect of SBWMV, which was distributed patchily in the 2007 experiment, may have played a role, although there may have been other factors as well. In any case, the longest duration of misting, 30 days, corresponds to a number of moist days that would be rare between wheat anthesis and harvest in the southeastern United States. For practical purposes, increasing numbers of wet days after flowering are likely to be associated with increased disease symptoms. Longer durations of post-anthesis moisture are thus likely to result in yield and test weight losses (22) as well as increases in grain DON. Of course, because all mist treatments started on the same date, we do not know if our results would have been the same had the shorter mist durations been initiated later in the grain-fill period.

Why did we find a positive association between number of post-anthesis moist days and disease and DON, while Culler et al (4) and Lemmens et al (11) found negative associations? The likeliest explanation seems to be that they were testing a higher moisture range, both in terms of numbers of wet days and moisture per day. Culler et al. compared 15 to 16 days with 31 to 32 days of post-flowering mist, and were delivering $63 \mathrm{~min}$ and $3.6 \mathrm{~mm}$ of moisture per day, with 14 mist periods over a 14-h period. During the inoculation-to-harvest period, they had a total of $248 \mathrm{~mm}$ of rain in 2002 and $214 \mathrm{~mm}$ of rain in 2003 (estimated from Figure 1 in Culler et al. [4]). Thus, their shorter misting treatment (15 to 16 days) resulted in totals of 302 and $272 \mathrm{~mm}$ of

TABLE 5. Percent infected kernels in soft red winter wheat cultivars planted in Kinston, NC, field experiment on the effects of post-anthesis moisture on Fusarium head blight (FHB) severity and deoxynivalenol (DON) accumulation

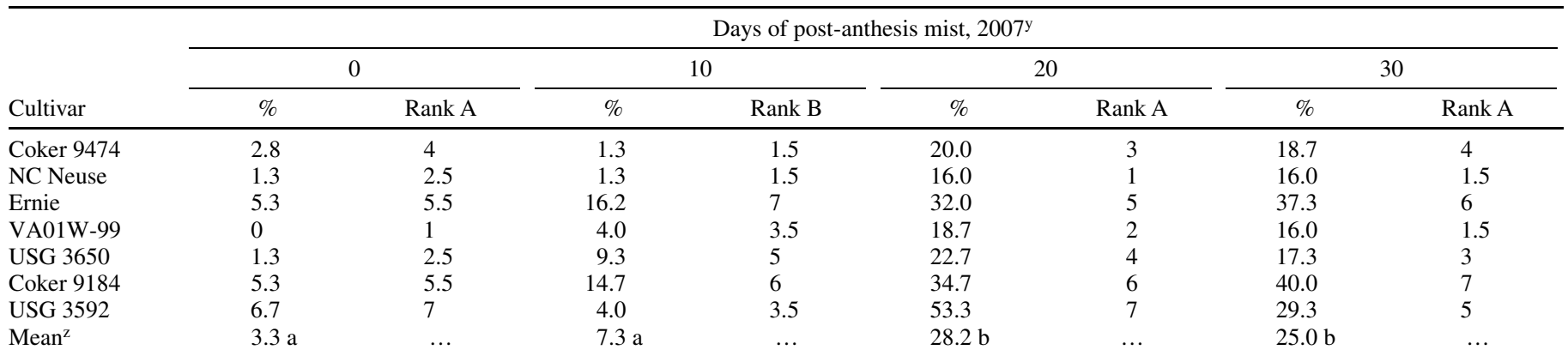

${ }^{y}$ Mist durations whose rank column headings are followed by the same uppercase letter did not rank cultivars differently at $P \leq 0.05$ by Spearman's rank correlation test. Lower rank numbers indicate lower percentages of infected kernels. Percentage of kernels infected by Fusarium graminearum (\%) measured using Komada's selective medium.

${ }^{\mathrm{z}}$ Means followed by the same letter are not different at $P \leq 0.05$ by least-squares means.

TABLE 6. Deoxynivalenol (DON) concentrations (Conc) in grain of soft red winter wheat cultivars planted in Kinston, NC, field experiment and subjected to different durations of post-anthesis mist ${ }^{\mathrm{x}}$

\begin{tabular}{|c|c|c|c|c|c|c|c|c|c|c|c|c|c|c|c|c|}
\hline \multirow[b]{3}{*}{ Cultivar } & \multicolumn{8}{|c|}{ Days of post-anthesis mist, 2006} & \multicolumn{8}{|c|}{ Days of post-anthesis mist, 2007} \\
\hline & \multicolumn{2}{|c|}{0} & \multicolumn{2}{|c|}{10} & \multicolumn{2}{|c|}{20} & \multicolumn{2}{|c|}{30} & \multicolumn{2}{|c|}{0} & \multicolumn{2}{|c|}{10} & \multicolumn{2}{|c|}{20} & \multicolumn{2}{|c|}{30} \\
\hline & Conc & Rank A & Conc & Rank B & Conc & Rank C & Conc & Rank C & Conc & Rank A & Conc & Rank AB & Conc & Rank B & Conc & Rank B \\
\hline NC Neuse & 2.1 & 1.5 & 6.7 & 5 & 8.3 & 1 & 10.2 & 4 & 1.8 & 5 & 1.6 & 3.5 & 4.3 & 3.5 & 2.0 & 2 \\
\hline Ernie & 2.7 & 3 & 3.8 & 1 & 14.7 & 8 & 13.3 & 7 & 2.8 & 6 & 2.6 & 6 & 4.3 & 3.5 & 3.3 & 5 \\
\hline VA01W-99 & 3.6 & 4 & 6.3 & 3 & 11.2 & 5 & 12.8 & 6 & 0.7 & 2 & 1.6 & 3.5 & 3.0 & 2 & 2.1 & 3 \\
\hline V-Tribute ${ }^{y}$ & 4.4 & 5 & 5.7 & 2 & 9.5 & 3 & 8.5 & 2 & & $\ldots$ & $\ldots$ & $\ldots$ & $\ldots$ & $\ldots$ & $\ldots$ & $\ldots$ \\
\hline USG 3592 & 12.3 & 8 & 14.7 & 8 & 14.5 & 7 & 19.0 & 8 & 1.4 & 4 & 2.5 & 5 & 7.7 & 6 & 3.8 & 6 \\
\hline $\operatorname{Mean}^{\mathrm{z}}$ & $4.9 \mathrm{a}$ & $\ldots$ & $8.0 \mathrm{~b}$ & $\ldots$ & $11.6 \mathrm{c}$ & $\ldots$ & $12.0 \mathrm{c}$ & $\ldots$ & $1.7 \mathrm{a}$ & $\ldots$ & $2.4 \mathrm{~b}$ & $\ldots$ & $5.0 \mathrm{c}$ & $\ldots$ & $2.7 \mathrm{~b}$ & $\ldots$ \\
\hline
\end{tabular}

${ }^{\mathrm{x}}$ Concentrations given in micrograms per gram, equivalent to parts per million; rank signifies DON ranking of the cultivar within that column, with lower rank numbers indicating lower DON concentration. Within a year, two mist durations whose rank column headings are followed by the same uppercase letter did not rank cultivars differently at $P \leq 0.05$ by Spearman's rank correlation test.

${ }^{\mathrm{y}}$ V-Tribute was not planted in 2007 due to a seed mix-up.

${ }^{\mathrm{z}}$ Within a year, means followed by the same letter are not different at $P \leq 0.05$ by least squares means. 
moisture in the 2 years, respectively. They compared that with their extended moisture treatment ( 31 to 32 days), which resulted in totals of 360 and $329 \mathrm{~mm}$ in the 2 years, respectively. By contrast, we misted for $36 \mathrm{~min}$ or $9.9 \mathrm{~mm}$ per day. There were 82.3 and $33.3 \mathrm{~mm}$ of inoculation-to-harvest rain in our 2 years, respectively. Adding mist irrigation to that, our plots received totals of $82,181,280$, or $379 \mathrm{~mm}$ of water between inoculation and harvest in the first year, and 33, 132, 231, or $330 \mathrm{~mm}$ in the second year. Moreover, Culler et al. (4) utilized 14-h mist periods that included the night, when evaporation is less, whereas our misting occurred entirely between the hours of 0800 and 1700, with a 2-h break in the middle of that interval.

Lemmens et al. (11) compared a nonmisted block with a block misted every other day over a 42-day period for $24 \mathrm{~min} /$ day $(20 \mathrm{~s}$ every $15 \mathrm{~min}$ for $18 \mathrm{~h}$ per day, from 1600 to $1200 \mathrm{~h}$ the following day). Their report does not give a rate in millimeter per day. During the 16-day inoculation period, they recorded 37 and $109 \mathrm{~mm}$ of precipitation at the two locations, respectively; rainfall between inoculation and harvest was not reported.

In summary, our range of misting durations included shorter intervals than those used in the other two studies, and the total amount of moisture in our experiment was substantially less than that in Culler et al (4). The shortest post-anthesis misting interval evaluated by Culler et al. was 15 days, whereas we had a 0-day treatment, and Culler et al. applied a much greater duration and larger daily volume of moisture than we did. Lemmens et al. (11) did have a nonmisted treatment but compared it only with a 42-day period of misting on alternate days, whereas we had intermediate durations of 10 and 20 days and a maximum of 30 days.

In fact, our data suggest a peaking somewhere between 20 and 30 days after anthesis of the elevating effect on DON of moisture duration. This is compatible with the idea (suggested by Culler et al. [4]) that numbers of moist days and volumes of water beyond those tested in our study might leach DON, resulting in lower DON levels relative to nonirrigated treatments. Of course, it should also be noted that the three studies occurred in different regions of the world (Minnesota, North Carolina, and Austria) and the Minnesota study used spring rather than winter wheat.

Lemmens et al. (11) reported a significant interaction between host genotype and mist irrigation with regard to DON concen-

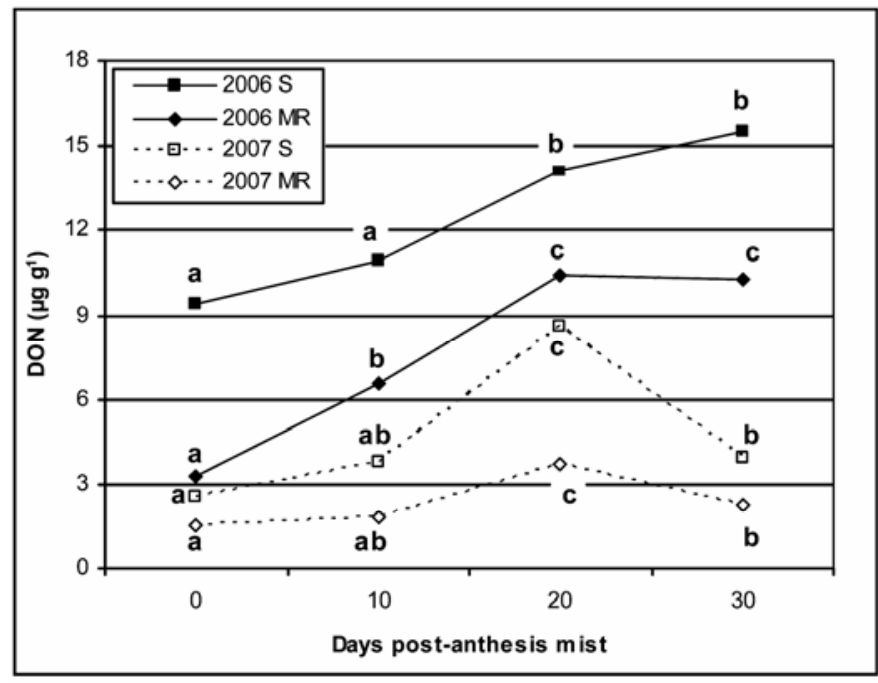

Fig. 2. Deoxynivalenol (DON) concentrations in wheat grain from a misted field nursery at Kinston, NC, in trials conducted in 2006, where data are means of six moderately resistant (MR) and two susceptible (S) winter wheat cultivars, and 2007, data are means of five MR and two S cultivars. Mist was applied post-anthesis for intervals of $0,10,20$, or 30 days, starting at midanthesis. Within a combination of year and resistance level (e.g., $2006 \mathrm{~S}$ ), data points with the same letter are not significantly different at $P \leq 0.05$. There were three replicate plots of each combination of cultivar and mist duration. tration (i.e., in some cases, a genotype had higher DON under irrigation than without it, whereas in other cases the effect was the opposite). We subjected the area under the disease progress curve (AUDPC) and DON data in Table 3 of Lemmens et al. (11) to Spearman rank correlation analysis. The result was that, in the lower-disease environment (location 1), there was no significant change in AUDPC ranking of cultivars with irrigation versus without irrigation $(\rho=0.95, P<0.0001)$. However, there was a significant irrigation-associated change in AUDPC ranking in the higher-disease environment (location $2 ; \rho=0.47, P=0.17$ ). In both environments, irrigation significantly changed the way cultivars were ranked for DON $(P \geq 0.16)$ and, in the higherdisease environment, the rank correlation coefficient $\rho$ was negative $(-0.48)$, whereas in the lower-disease environment it was positive $(0.47)$.

Our result is consistent with that of Lemmens et al. (11). In the higher-disease and higher-DON environment (2006) of our study, there was a greater change in ranking for DON associated with increasing moist days than in our lower-disease environment (Table 6). Cv. Ernie, for example, lost its low DON rank in 2006 under 20 and 30 days of post-anthesis mist, while in the lowerDON year of 2007, it actually improved its DON rank under the 20-day mist duration. These ranking changes may reflect genetic differences among cultivars in ability to resist DON development under increasing moisture durations. In other words, the higher the disease pressure in a nursery, the greater may be the differential effects of varying post-anthesis moisture durations on cultivars' DON rankings. In practical terms, it may be helpful to subject the most DON-resistant germplasm to longer durations of post-anthesis moisture to see if these materials maintain their low DON rank.

None of the cultivars in our experiment appeared to possess the alleles for resistance present in Chinese germplasm such as Sumai 3 or Wuhan 1. Among the cultivars in this experiment were some of the most Fusarium-resistant cultivars adapted to the southeastern United States, such as Ernie, Coker 9474, and NC Neuse. These cultivars have high levels of partial resistance to initial infection or spread within the head derived from non-Chinese sources within regional breeding stocks. They represent germplasm widely grown or crossed with in this region. Further, our results appeared to apply equally to moderately resistant cultivars with either type I or type II resistance, or both, and to susceptible cultivars. Thus, our results are likely applicable to breeding programs in this region, if not beyond.

This study involved infections occurring due to artificial inoculation on a single day at mid-anthesis. Why, then, did DI vary with post-anthesis mist duration? The answer is not likely to be secondary infections because, for the most part, there would not have been time for originally infected heads to form sporodochia for polycyclic infections. Perhaps some $F$. graminearum spores remained on the surface or in crevices of glume tissues, rather than infecting anthers, and their ability to eventually penetrate the glumes was affected by the duration of moist conditions. Ambient inoculum from external sources may also have had greater success at causing late infections in plots with post-anthesis mist, although it is doubtful that this explained all of the DI increase in those plots, because border plots did not exhibit similarly elevated disease levels.

Although DS was similar in the 2 years, DI was higher in 2006 than in 2007 (Fig. 1). Evidently, there was a lower infection efficiency in 2007 than in 2006, likely due in large part to a greater variance in flowering timing associated with SBWMV in the FHB nursery. However, post-anthesis mist allowed the fungus to eventually spread within spikes that were infected in 2007 to the same extent in terms of percent bleached spikelets as in 2006. Indeed, under 0 days of misting DS was twice in 2006 what it was in 2007; however, the ratio changed to one of equality by 20 days of misting. This suggests that extended post-anthesis 
moisture can compensate for a scarcity of infections at anthesis by elevating DS.

However, mean DON levels with 0 days of mist were over twice in 2006 what they were in 2007 and, although they increased above the FDA-recommended threshold of $2 \mu \mathrm{g} / \mathrm{g}$ for human consumption under 10 days of mist, even with 20 days of mist they were still twice in 2006 what they were in 2007 (Table 6 ). In the case of FDK, the most direct measure in our experiment of effects upon yield and test weight, it took the two longest durations of post-anthesis mist to produce significant increases in FDK percentages, and mean levels in the lower-DI year of 2007 did not approach those in the higher-DI year under any mist duration (Table 4). Taken together, these results suggest that the aggravating impact of post-anthesis moisture is most evident with respect to visual disease symptoms, although it also worsens kernel damage and DON. Our results qualify the suggestion of Schaafsma et al. (8) that "there is usually little concern of excessive DON in grain with dry conditions during anthesis."

Our results have obvious implications for disease and DON prediction models. For DON forecasting, the number of wet days after anthesis is a factor that must be taken into account along with other relevant environmental variables, such as pre-anthesis precipitation and temperature. From a grower's perspective, prolonged rainy periods following wheat flowering should be a signal to scout wheat crops for FHB symptoms, even if pre-flowering conditions were not conducive to FHB.

As discussed above, the implications of our findings for breeding programs should be considered. Many breeders now assess cultivars for FHB resistance based on either natural rainfall or misting during the period around anthesis. Our data suggest that cultivar rankings for several indicators commonly used by breeders (DI, DS, FDK, and DON) may change under extended post-anthesis irrigation.

\section{ACKNOWLEDGMENTS}

This research was supported by a grant from the U.S. Wheat \& Barley Scab Initiative, and we also thank the North Carolina Small Grain Growers Association for financial support. We thank D. Fulbright, P. Hart, and C. Medina-Mora, Michigan State University Regional Diagnostic Center for FHB, and Y. Dong, University of Minnesota, for their DON testing services; and E. Duren, M. Fountain, D. Fulbright, E. Hrebenuyuk, P. Langdon, J. Lovett, L. Martin, R. Parks, R. Peeler, A. Perry, W. Xu, J. H. Yang, D. Yigit, and the staff of Cunningham Research \& Extension Center for excellent technical assistance.

\section{LITERATURE CITED}

1. Buerstmayr, H., Steiner, B., Schmolke, M., Schondelmeier, J., Scholz, U., Wilde, F., Miedaner, T., Ebmeyer, E., Korzun, V., Ruckenbauer, P., and Lemmens, M. 2004. QTLs and markers for FHB resistance in wheat. Page 26 in: Proc. 2nd Int. Symp. Fusarium Head Blight. S. M. Canty, T. Boring, J. Wardwell, and R. W. Wards, eds. Michigan State University, East Lansing.

2. Chen, J., Griffey, C. A., Maroof, M. A. S., Biyashew, R. M., Zhao, W., Chappell, M., and Dong, Y. 2004. Update on QTL mapping of Fusarium head blight resistance in wheat. Page 32 in: Proc. 2nd Int. Symp. Fusarium Head Blight. S. M. Canty, T. Boring, J. Wardwell, and R. W. Wards, eds. Michigan State University, East Lansing.

3. Cowger, C., and Sutton, A. L. 2005. The southeastern U.S. Fusarium head blight epidemic of 2003. Published Online. Plant Health Progress DOI: 10.1094/PHP-2005-1026-01-RS.

4. Culler, M. D., Miller-Garvin, J. E., and Dill-Macky, R. 2007. Effect of extended irrigation and host resistance on deoxynivalenol accumulation in Fusarium-infected wheat. Plant Dis. 91:1464-1472.

5. DeWolf, E. D., Madden, L. V., and Lipps, P. E. 2003. Risk assessment models for wheat Fusarium head blight epidemics based on within-season weather data. Phytopathology 93:428-435.
6. Doohan, F. M., Mentewab, A., and Nicholson, P. 2000. Antifungal activity toward Fusarium culmorum in soluble wheat extracts. Phytopathology 90:666-671.

7. Hart, L. P., Pestka, J. J., and Liu, M. T. 1984. Effect of kernel development and wet periods on production of deoxynivalenol in wheat infected with Gibberella zeae. Phytopathology 74:1415-1418.

8. Hooker, D. C., Schaafsma, A. W., and Tamburic-Ilincic, L. 2002. Using weather variables pre- and post-heading to predict deoxynivalenol content in winter wheat. Plant Dis. 86:611-619.

9. Kang, Z., and Buchenauer, H. 1999. Immunocytochemical localization of Fusarium toxins in infected wheat spikes by Fusarium culmorum. Physiol. Mol. Plant Pathol. 55:275-288.

10. Langevin, F., Eudes, F., and Comeau, A. 2004. Effect of tricothecenes produced by Fusarium graminearum during Fusarium head blight development in six cereal species. Eur. J. Plant Pathol. 110:735-746.

11. Lemmens, M., Buerstmayr, H., Krska, R., Schuhmacher, R., Grausgruber, H., and Ruckenbauer, P. 2004. The effect of inoculation treatment and long-term application of moisture on Fusarium head blight symptoms and deoxynivalenol contamination in wheat grains. Eur. J. Plant Pathol. 110:299-308.

12. Lemmens, M., Scholz, U., Berthiller, F., Koutnik, A., Dall'Asta, C., Schuhmacher, R., Adam, G., Mesterhazy, A., Krska, R., Buerstmayr, H., and Ruckenbauer, P. 2004. The role of resistance to deoxynivalenol in the complex Fusarium head blight resistance complex in wheat. Pages 88 in: Proc. 2nd Int. Symp. Fusarium Head Blight. S. M. Canty, T. Boring, J. Wardwell, and R. W. Wards, eds. Michigan State University, East Lansing.

13. McCartney, C. A., Somers, D. J., Fedak, G., and Cao, W. 2004. Haplotype diversity at Fusarium head blight QTLs in wheat. Theor. Appl. Genet. 109:261-271.

14. McMullen, M., Jones, R., and Gallenberg, D. 1997. Scab of wheat and barley: A re-emerging disease of devastating impact. Plant Dis. 81:13401348.

15. Mesterhazy, A. 1995. Types and components of resistance to Fusarium head blight of wheat. Plant Breed. 114:377-386.

16. Miedaner, T., Moldovan, M., and Ittu, M. 2003. Comparison of spray and point inoculation to assess resistance to Fusarium head blight in a multienvironment wheat trial. Phytopathology 93:1068-1072.

17. Mohammadi, M., and Kazemi, H. 2002. Changes in peroxidase and polyphenol oxidase activities in susceptible and resistant wheat heads inoculated with Fusarium graminearum and induced resistance. Plant Sci. 162:491-498.

18. Murphy, J. P., and Navarro, R. 2001. Uniform Southern Soft Red Winter Wheat Fusarium Head Blight Screening Nursery, 2001 Nursery Report. Crop Science Research Report No., North Carolina State University, Raleigh.

19. Murphy, J. P., Navarro, R., and Srnic, G. 2000. Uniform Southern Soft Red Winter Wheat Fusarium Head Blight Screening Nursery, 2000 Nursery Report. Crop Science Research Report No., North Carolina State University, Raleigh.

20. Murphy, J. P., Navarro, R. A., and Van Sanford, D. A. 2002. Uniform Southern Soft Red Winter Wheat Fusarium Head Blight Screening Nursery, 2002 Nursery Report. Crop Science Research Report No., North Carolina State University, Raleigh.

21. Murphy, J. P., Navarro, R. A., and Van Sanford, D. A. 2003. Uniform Southern Soft Red Winter Wheat Fusarium Head Blight Screening Nursery, 2003 Nursery Report. Crop Science Research Report No., North Carolina State University, Raleigh.

22. Parry, D. W., Jenkinson, P., and McLeod, L. 1995. Fusarium ear blight (scab) in small grain cereals-a review. Plant Pathol. 44:207-238.

23. Pritsch, C., Muehlbauer, G. J., Bushnell, W. R., Somers, D. A., and Vance, C. P. 2000. Fungal development and induction of defense response genes during early infection of wheat spikes by Fusarium graminearum. Mol. Plant-Microbe Interact. 13:159-169.

24. Rotter, B. A., Prelusky, D. B., and Pestka, J. J. 1996. Toxicology of deoxynivalenol (vomitoxin). J. Toxicol. Environ. Health 48:1-34.

25. Rudd, J. C., Horsley, R. D., McKendry, A. L., and Elias, E. M. 2001. Host plant resistance genes for Fusarium head blight: Sources, mechanisms, and utility in conventional breeding programs. Crop Sci. 41:620-627.

26. Schroeder, H. W., and Christensen, J. J. 1963. Factors affecting resistance of wheat to scab caused by Gibberella zeae. Phytopathology 53:831-838.

27. Siranidou, E., Kang, Z., and Buchenauer, H. 2002. Studies on symptom development, phenolic compounds and morphological defence responses in wheat cultivars differing in resistance to Fusarium head blight. J. Phytopathol. 150:200-208. 